\title{
Les Représentations Schématiques Des Enseignants Stagiaires En Sciences De La Vie Et De La Terre : Quelles Compétences Pour Une Utilisation Pédagogique?
}

\author{
Hasnae El Hnot (PhD), Assistant Prof. \\ Bouamama Cherai (PhD), Assistant Prof.
}

Département des Sciences de la Vie et la Terre, Centre Régional des Métiers de l’Éducation et de la Formation (CRMEF), Tanger - Tétouan - Al

Hoceima, Maroc.

\section{Hayat Sibari (PhD), Assistant Prof.}

Département des Sciences de la Vie et la Terre, Centre Régional des Métiers de l'Éducation et de la Formation (CRMEF), Rabat - Salé- Kénitra, Maroc.

doi: 10.19044/esj.2017.v13n19p211 URL:http://dx.doi.org/10.19044/esj.2017.v13n19p211

\begin{abstract}
This article aims to analyze schemes made by teacher trainees in Life and Earth sciences at the Regional Center of the Trades of Education and Training (CRMEF) Tangier-Tetouan-Al Houceima- middle school - in order to identify the conceptions of these future teachers about the schematic representation of a concept.

An analysis was made of 30 teacher trainees diagrams at the beginning of their qualifying training on concepts in internal geodynamics. The results obtained have shown that they are generally known by teacher trainees, but their schematic representations raise many problems, notably the scientific aspect of the scheme, the maintenance of a correct methodological organization and the respect for the symbolic code to be done on a scientific basis. To remedy this, the qualifying training of these teacher trainees must be reinforced with schematization sessions supplemented by an integration of these schemes in sequences of learning for better pedagogical use.
\end{abstract}

Keywords : Schemes, geology, teacher trainees, scientific concepts, CRMEF, Morocco

Résumé

Cet article a pour objectif d'analyser des schémas faits par des enseignants stagiaires en Sciences de la vie et de la terre au Centre Régionale 
des Métiers de l'Éducation et de la Formation (CRMEF) Tanger-Tétouan-Al Houceima - cycle collégial - afin de relever les conceptions qu'ont ces futurs enseignants concernant la représentation schématique d'un concept.

Une analyse a été faite auprès de 30 schémas des enseignants stagiaires en début de leur formation qualifiante portant sur des concepts en géodynamique interne. Les résultats obtenus ont mis en évidence que ces derniers sont globalement connus par les enseignants stagiaires mais leurs représentations schématiques posent beaucoup de problèmes notamment l'aspect scientifique du schéma, le maintien d'une organisation méthodologique correcte et le respect du code symbolique qui doit être fait sur des bases scientifiques. Pour remédier à cela la formation qualifiante de ces enseignants stagiaires doit être renforcée de séances de schématisation complétée par une intégration de ces schémas dans des séquences d'apprentissage pour une meilleure utilisation pédagogique.

Mots-clés : Schémas, géologie, enseignants stagiaires, concepts scientifiques, CRMEF, Maroc

\section{Introduction}

L'enseignement des sciences en général, et celui des sciences de la vie et de la terre (SVT), en particulier se base sur des démarches scientifiques qui permettent de développer chez l'apprenant un esprit scientifique basé sur l'observation et sur l'analyse, c'est pourquoi il utilise largement tous les systèmes de représentations : schémas, croquis, tableaux, graphiques.... L'utilisation de ce langage symbolique donne à ces productions des pouvoirs organisateurs et structurants particuliers par rapport aux savoirs et aux observations scientifiques (Cooper et Destin, 2015). Les représentations graphiques jouent un rôle bien plus important qu'apporter une simple illustration, ils fournissent des informations spécifiques qu'un texte, par son côté linéaire, ne peut livrer aussi efficacement. En outre, en permettant une visualisation des données, ils offrent des possibilités de structuration et de clarification des informations (Giot et al., 2006 ; Giot et Quittre, 2008).

Comme chacun de ces supports visuels, les schémas apportent un soutien certain à la pensée scientifique et constituent une forme très spécifique de langage communicationnel. Ils fournissent des référentiels spatialement et cognitivement économiques et permettent de visualiser l'information et de reformuler la pensée en la structurant (Giot et Quittre, 2008 ; 2011). Aussi, les schémas en centrant sur l'essentiel, permettent non seulement un meilleur apprentissage des connaissances présentées mais, conduisent au-delà d'une capacité de reproduction de ce qui vient d'être appris, à une capacité de transfert à d'autres données. Le schéma devient 
alors un moyen de communication parmi les autres moyens utilisés, chacun répondant à un objectif qui lui est propre (Vezin, 1986).

En didactique des SVT, le schéma prend une dimension importante dans l’apprentissage. Il occupe ainsi une place importante dans la construction de compétences communicatives chez l'apprenant. En effet, l'utilisation de schémas dote l'enseignant au cours de son activité d'une véritable palette de moyens didactiques, tout en offrant une grande plasticité d'interprétations et d'utilisations au développement du discours enseignant, à condition qu'il sache s’en servir (Buty et Badreddine, 2009).

« Schématiser » n’est pas un savoir-faire inné chez le jeune enfant, il nécessite des connaissances, des capacités et la maîtrise d'un certain langage scientifique ; c'est pourquoi il doit faire l'objet d'un apprentissage spécifique et progressif qui doit s'adapter à l'âge et au niveau du public (Cooper et Destin, 2015). Toutefois, ils engendrent des difficultés parfois importantes. Ainsi, pour utiliser ce support scientifique, l'élaborer et bien l'exploiter et l'intégrer dans une séquence d'apprentissage les enseignants doivent bien maitriser cet outil et pouvoir développer cette compétence chez l'apprenant en adoptant des orientations méthodologiques adéquates pour étayer efficacement la construction de la pensée.

Dans ce sens, et vu l'importance d'acquisition de l'enseignant des compétences nécessaires pour la construction et l'intégration des schémas dans les séquences d'apprentissage, nous centrons notre recherche sur les enseignants stagiaires, en formation qualifiante en SVT, au Centre Régional des Métiers de l'Education et de la Formation (CRMEF) au cycle collégial. En effet, les orientations pédagogiques de ce cycle placent la schématisation au sein de compétences communicatives que doit acquérir l'élève et qui doit être développées chez l'apprenant sous différentes formes écrite, orale, schématique... (MEN, 2009).

Ainsi, et dans le cadre de notre recherche, nous avons choisi de mettre l'accent sur les représentations schématiques des enseignants stagiaires de quelques concepts en géodynamique interne et donc de nous intéresser aux savoirs scientifiques qu'ont ces enseignants stagiaires et leur capacité de transmettre ces savoirs à travers des schémas simples expliquant un phénomène scientifique en général et géologique en particulier.

L’objectif principal de cette étude est de savoir les conceptions des enseignants stagiaires envers les représentations schématiques d'un phénomène géologique à partir de l’analyse des différents schémas réalisés. Pour cela nous cherchons à répondre aux questions suivantes :

- Comment expliquer un phénomène géologique en le représentant schématiquement?

- Quelles sont les données fournies par les schémas sur les connaissances et les savoirs faire des enseignants stagiaires ? 
- Quels sont les obstacles rencontrés par les futurs enseignants lors de la réalisation des schémas scientifiques en géologie?

\section{Cadre théorique}

\section{Schémas : définition et caractéristiques}

Le schéma est défini comme une représentation simplifiée qui permet de mettre en relief non la forme, mais les relations, voire le fonctionnement des éléments essentiels d'un objet ou d'un processus pour faciliter la compréhension de sa composition, de son fonctionnement, et de son organisation. Cette représentation permet d’organiser des données les unes par rapports aux autres pour communiquer un message à quelqu'un qui en fait une lecture interprétative (Chantal, 2010 ; Cooper et Destin, 2015). Les schémas peuvent, ainsi, traduire des concepts, des relations, des phénomènes ou des fonctionnements, et leur complexité varie en fonction de ces objets d'étude (Giot et Quittre, 2011).

Les schémas constituent une forme importante d'information visuelle et exigent des capacités d'interprétation spécialisées (Lowe, 1996). Ils favorisent la sélection de 1'information et attirent l'attention sur l'essentiel et sur l'interaction des données à apprendre. Ils ont une valeur de généralité et ne représentent que les caractéristiques générales valables pour une catégorie d'objets ou de phénomènes (Vezin, 1986). Selon cet auteur, les schémas ont des fonctions qui leur sont propres et qui répondent, dans une situation pédagogique, à des objectifs bien précis et non à la simple volonté de faire mieux apprendre. Ainsi, en fonction de ces objectifs, les schémas se caractérisent par quatre points essentiels dont la connaissance accompagne leur utilisation (Le plat et Hoc, 1983 ; Peraya, 1995 ; Peraya et Nyssen, 1995 ; Astolfi, 2001):

- une capacité à isoler des relations invariantes au sein d'un ou de plusieurs phénomènes ;

- une capacité à concrétiser des concepts ou des phénomènes difficiles à représenter;

- $\quad$ une valeur synoptique par utilisation d'espace pour présenter sur un même document des éléments essentiels ;

- $\quad$ une économie cognitive en diminuant la charge mnémonique et en la faisant porter sur l'essentiel.

Les schémas possèdent un langage propre par la présence de différents outils : les icônes (symboles figuratifs ou abstraits), les signes (flèches ; traits...), les annotations (titres, légendes, commentaires), l'échelle, les proportions et les positions relatives des éléments, la perspective (coupe, plan, dessin en 3D), les couleurs... Le choix des icônes, des couleurs et des signes peut être propre à l'auteur, usuel ou conventionnel (Giot et Quittre, 2011). L'utilisation de ce langage symbolique confère à ces productions des 
pouvoirs organisateurs et structurants particuliers par rapport aux savoirs et aux observations scientifiques, toutefois, la sélection d'informations, l'abstraction, ne se fait pas au hasard : elle répond au besoin de construire un concept (Oillic, 2014 ; Cooper et Destin, 2015).

La construction d'un schéma dépend, en premier lieu, de la détermination de l'intention de celui qui produit le document. C'est à partir de cette intention qu'il est possible de sélectionner les informations qui vont la traduire (Langellier, 2004 ; Oillic, 2015). Ainsi, le schéma prend plusieurs formes selon les différentes intentions qui motivent cette construction (Giot et Quittre, 2011):

- $\quad$ accrocher, motiver, attirer l'attention du lecteur ;

- fournir un référentiel, une représentation substitutive en remplacement d'un réel qui ne peut être introduit en classe ;

- $\quad$ décrire, faire visualiser ;

- $\quad$ organiser et agencer spatialement des données ;

- créer ou construire une réflexion en facilitant la mise en forme d'idées, et en élaborant des modèles en relation avec la perception qu'on peut avoir du réel ;

- imaginer pour stimuler la mémorisation, encourager la compréhension et impliquer plus affectivement le lecteur.

Les caractéristiques des schémas, leurs outils et les intentions de celui qui les produit impliquent des interprétations de la part du lecteur, d'autant plus que le symbolisme et les modalités de structuration des schémas présentent une grande variabilité ; c’est pourquoi, il est indispensable de prendre en compte plusieurs indices pour comprendre l'ensemble d'un schéma (Giot et Quittre, 2011).

\section{Intégration des schémas dans l’apprentissage}

Le schéma est un outil didactique utilisé pour diverses situations d'apprentissages ou pour des phases de structuration des connaissances (Cooper et Destin,; 2015). Monnier (2003), dans son travail apprécie l'apport des schémas dans le cadre de la résolution de problèmes et retient que les schémas peuvent être une aide à la construction de la représentation du problème, mais encore faut-il varier les schémas et être attentif au mode d'apprentissage proposé et à l'acquisition ou non de ces schémas par les élèves. Buty et Badreddine (2009) soulèvent le lien entre le rôle structurant de l'usage de schémas et sa manière d'intégration dans le discours scientifique. Ainsi, les schémas, tout en incorporant des savoirs et des contraintes, offrent une grande plasticité d'interprétations et d'utilisations au développement du discours enseignant : ils représentent ce qu'on admet, et clarifient ce qui doit être discuté. Toutefois, l’apprentissage ne se situe pas 
dans le schéma, mais dans le discours de la communauté de la classe dans lequel il prend son sens. Ainsi selon Lowe (1996), il est parfaitement possible, pour un étudiant, d'apprendre plus d'une discussion bien conçue, conduite par un enseignant autour d'un schéma statique, que d'une proposition multimédia d'animation interactive dépourvue de réflexion didactique.

Giot et quittre (2008) montrent qu'il ne suffit pas de simplifier un schéma pour le rendre plus compréhensible. Il s’agit plutôt de permettre aux élèves d'acquérir progressivement les rudiments du langage iconique en sciences. C'est pour cela que les activités de symbolisation, de reformulation et de transcodage doivent occuper une place importante et éviter l'imposition de savoirs magistraux (Astolfi et al., 1989). Ainsi, il est intéressant de faire percevoir à l'élève toute la portée communicationnelle du schéma et le mettre en contact progressif et régulier avec ces supports au sein d'activités scientifiques significatives. En effet, c’est souvent grâce à de telles activités que devient possible la transformation des représentations conceptuelles en reconstitutions plus conforme aux exigences scientifiques (Giot et Quittre, 2011 ; Oillic, 2014 ; Cooper et Destin, 2015). En effet, en privilégiant les formes et les couleurs, le schéma permet à l'élève de communiquer sa réflexion et de représenter certaines notions compliquées plutôt que de les expliquer par un long texte et sans être bloqué par un manque de vocabulaire.

Enfin, pour que le schéma soit un véritable apport, pour qu'il soit compris et qu'un travail de réflexion puisse être opéré au dessus, l'élève devra aborder chaque étape de sa construction en mobilisant ses capacités d'abstraction et de modélisation, et en optimisant sa compréhension et sa mémorisation (Oillic, 2014).

\section{Méthodologie de recherche}

Le travail présenté ici s’intéresse particulièrement aux représentations schématiques, réalisées par les enseignants stagiaires, de certains concepts géologiques. C'est ainsi que nous avons eu recours à l'analyse de schémas en vue de chercher les difficultés et les obstacles que peuvent avoir nos candidats face à une représentation schématique.

Pour cela nous nous sommes basés sur un test distribué aux enseignants stagiaires des SVT du CRMEF de Tanger au début de l'année de formation 2015-2016. L'élaboration de ce test vient dans le cadre d'une évaluation diagnostique en début du module de géodynamique interne. Notre test comprend plusieurs outils : des questions ouvertes, fermées, lacunes à remplir... Parmi les questions posées, celle qui a fait l'objet de cet article : "Réaliser un schéma simple résumant le phénomène de mise en place d'un granite et son impact sur les roches encaissantes, sachant que ces dernières 
sont affectées par une faille et un plissement antérieur à l'intrusion granitique »

Nous avons précisé comme tache de schématiser trois éléments principaux qui sont "le granite, les plis et la faille". En effet, ces derniers font objet d'apprentissage dans le programme des SVT de la deuxième année du cycle collégial. Ils sont intégrés dans l'unité de géodynamique interne au sein des parties portant sur les déformations tectoniques cassantes et souples et sur les roches magmatiques et sont systématiquement liés dans une approche de compréhension globale des phénomènes de la géodynamique interne.

Pour mieux analyser l’agencement de ces différents éléments dans un schéma nous avons précisé que les terrains traversés par l’intrusion granitique sont faillés et plissés au cours d'une déformation antérieure. Les schémas récoltés ont été énumérés, aléatoirement, de S1 à S30.

Afin de faire une corrélation entre les schémas réalisés par les enseignants stagiaires et leurs profils, nous avons élaborés des fiches de renseignements qui permettent de définir le profil des enseignants stagiaires, leur parcours universitaire, leur besoin en formation... L'utilisation de ces données est visée par la formation et ultérieurement par cette recherche.

\section{Population cible}

Notre recherche a été réalisée auprès d'un échantillon de 30 enseignants stagiaires des SVT du CRMEF de Tanger. Nous retrouvons dans le Tableau 1 les caractéristiques de la population cible.

\begin{tabular}{|c|c|c|c|c|c|}
\hline $\begin{array}{c}\text { Répartition par } \\
\text { type de licence }\end{array}$ & \multicolumn{2}{|c|}{$\mathrm{LPQME}^{*}=17(56,67 \%)$} & $\begin{array}{c}\mathrm{FUE}^{*}=11 \\
(36,67 \%)\end{array}$ & $\begin{array}{c}\mathrm{LF}^{*}=2 \\
(6,66 \%)\end{array}$ \\
\hline $\begin{array}{c}\text { Répartition par } \\
\text { spécialité }\end{array}$ & Géologie =6 & $\begin{array}{c}\text { Biologie }= \\
6\end{array}$ & $\begin{array}{c}\text { Écologie }= \\
5\end{array}$ & SVT =11 & Biologie =2 \\
\hline $\begin{array}{c}\text { Obtention d'un } \\
\text { Master }\end{array}$ & $\begin{array}{c}\text {-Géologie }=2 \\
\text {-Science de } \\
\text { l'éducation=1 }\end{array}$ & Biologie=2 & & & \\
\hline
\end{tabular}

Tableau 1 : Population cible de l'étude

LPQME : Licence Professionnelle de qualification aux métiers de l'enseignement ; LF :

Licence Fondamentale ; FUE : Licence de filière universitaire éducative.

Notre population cible est représentée par 3 types de diplôme : une Licence Professionnelle de Qualification aux Métiers de l'Enseignement (LPQME) qui représente 56,67\%, une licence de Filière Universitaire Éducative (FUE) représentée par 36,67\% et une Licence Fondamentale (LF) avec $6,66 \%$. Les différentes spécialités sont réparties comme suit: 6 géologie; 8 biologie; 5 écologie et 11 SVT, représentés par la filière éducative (FUE). 


\section{Résultats et discussion}

En analysant les productions des enseignants stagiaires, nous pouvons comprendre les stratégies cognitives mises en place dans l'organisation et l'agencement spatial des différents éléments schématisés. Nous ne chercherons pas à faire un inventaire des productions mais plutôt d'analyser les pensées qu'elles reflètent.

Notre analyse prendra en compte les éléments essentiels du schéma qui sont le granite ; la faille; le plissement et les roches encaissantes puis leurs dispositions les uns par rapport aux autres, l'usage du code symbolique et enfin une corrélation des schémas réalisés par les enseignants stagiaires et leurs profils.

\section{Représentations schématiques des éléments clés}

\section{L'élément 1 : le granite}

Nous nous sommes intéressés d'abord à la forme que prend le granite dans ces schémas. Nous pouvons regrouper ces schémas en 4 catégories (Figure 1) :

- Le premier qui est majoritaire et qui représente $70 \%$ montre un granite en forme concave étroite ou légèrement ouverte (S6).

- $\quad$ Le deuxième type qui représente $16,67 \%$, montre une intrusion magmatique sous forme d'un espace fermé prenant la forme d'un cercle ou d'un triangle (S18).

- $\quad$ Le troisième type (10\%) est représenté sous une forme toujours concave mais prenant une forme arrondie qui se referme un peu vers le bas (S5).

- $\quad$ Un seul schéma (3,33\%) montre un granite qui traverse des terrains rocheux sous forme d'un canal rectiligne ouvert en haut et en bas et qui semble communiquer avec l'extérieur (S28). Cette intrusion granitique semble n'avoir aucun lien chronologique avec les roches environnantes puisque le canal granitique n'est pas mis en valeur en étant antérieure ou postérieure à la déformation tectonique.

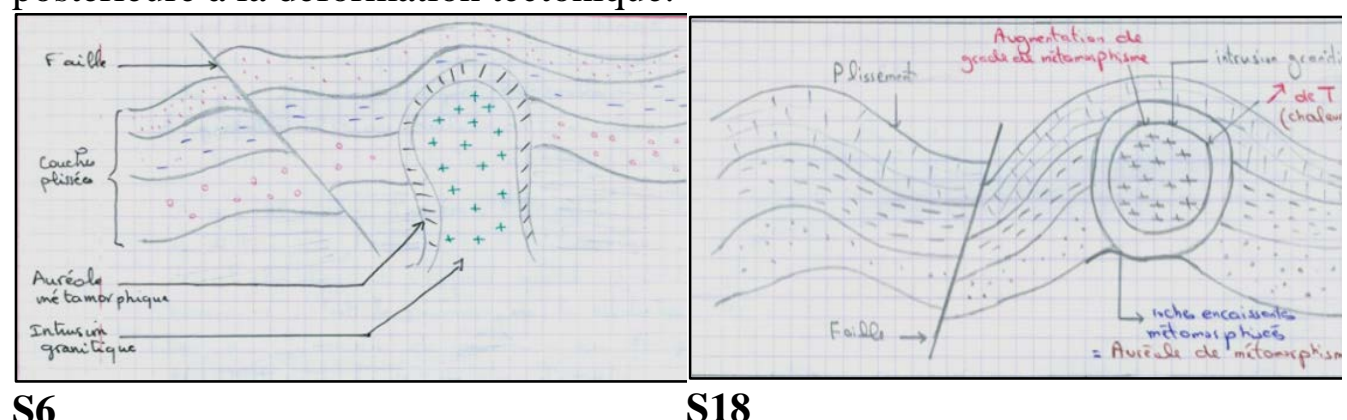




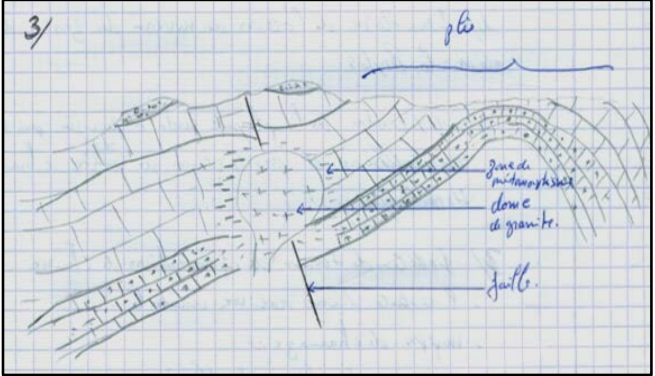

S5

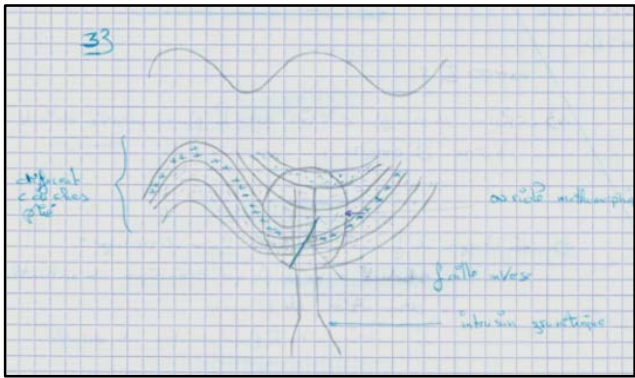

S28

Figure 1 : Les différentes formes de granite représenté dans les schémas des enseignants stagiaires

Concernant le code symbolique utilisé pour le granite, $87 \%$ des schémas présentent le granite avec le symbole $(++)$ désignant les roches acides, $10 \%$ des schémas ne présentent aucun symbole, et 3\% des schémas montrent le granite avec des croix (xx) symbole qui est réservé normalement aux roches volcaniques basiques (Figure 2).

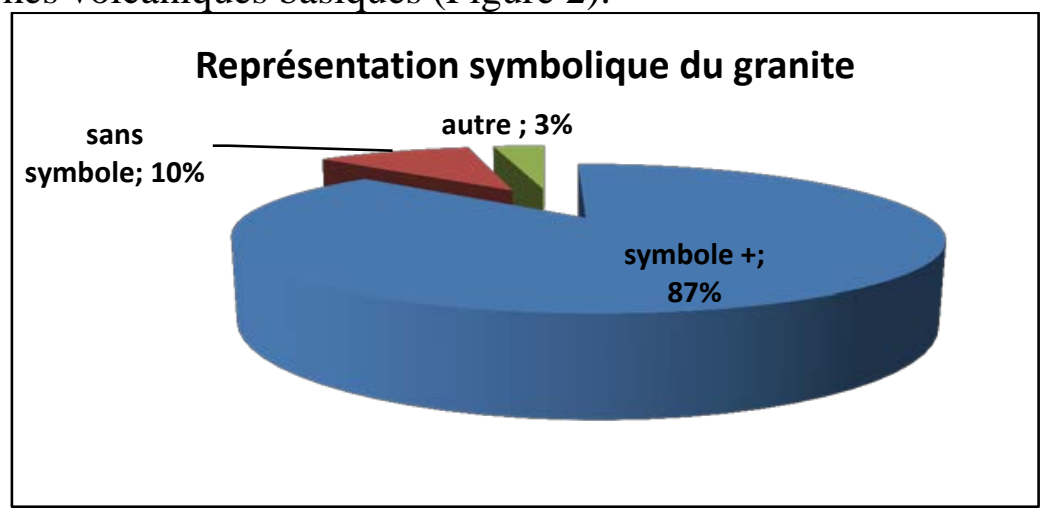

Figure 2 : Pourcentage de représentation du code symbolique du granite

Pour compléter cette analyse de l'élément granite, nous avons vu comment cette intrusion granitique est liée avec l'extérieur et principalement avec la profondeur (Figure 3). Nous nous retrouvons avec $87 \%$ des schémas qui ne montrent aucune liaison du granite avec la profondeur et $13 \%$ qui marquent ce lien par des remontées de magma acide sous forme de gouttelettes (S3) ou sous forme de petites zones à aspect ovale ou en concaves fermées ou ouvertes d'un côté vers le haut ou vers le bas (S15,S16,S30). A noter que sur le schéma S30 les remontées magmatiques prennent la forme d'un triangle pointu fermé vers le haut et l'ensemble, représenté par le granite et les roches encaissantes, suit la même forme triangulaire. 


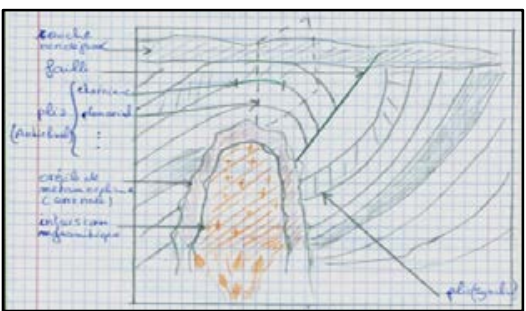

S3

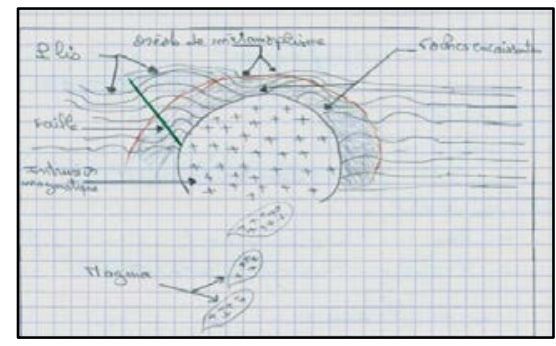

S16

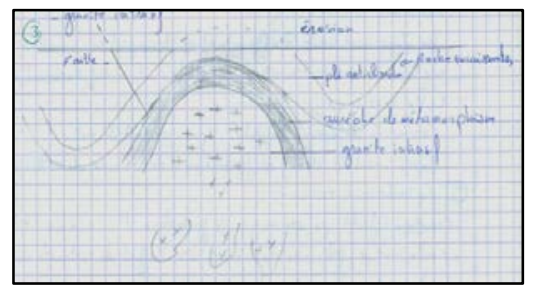

S15

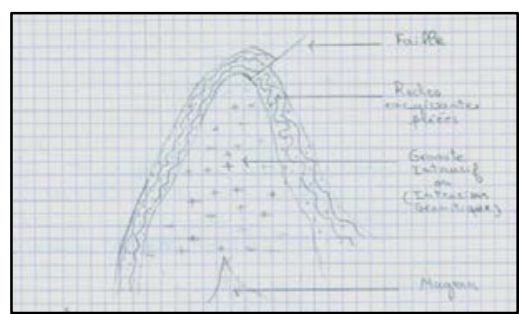

S30

Figure 3 : Représentation schématique de la relation granite-profondeur

L'analyse de l'élément granite nécessite aussi de voir comment est schématisée l'auréole de métamorphisme sur les 30 schémas étudiés. Nous avons ainsi relevé plusieurs manières de présenter cette auréole. Ainsi on retrouve sur 11 schémas une zone nette sous forme d'une bande à contour bien défini autour du granite, de couleur sombre (Figure 3,S3,S15); 9 schémas la montrent sous forme d'une zone à contour net ou diffus; à remplissage marqué par des symboles ou des figurés tels des points, des croix, des pointillés, des petits traits ou des traits obliques (Figure 1,S5,S6) et sur 6 schémas on retrouve un granite nu sans zone particulière qui le contourne (Figure 3,S30). Enfin, dans 3 schémas, l'auréole est montrée comme une zone vide à contour bien défini (Figure 1,S18) et un seul schéma la présente uniquement sous forme d'un cercle fin vide et à contour fin (Figure 1,S28).

\section{L'élément 2 : La faille}

La faille constitue sur ces schémas un élément primordial, c'est pourquoi nous avons tenu à voir comment elle est représentée. Notre analyse montre que $67 \%$ des représentations schématiques présentent un rejet de faille avec un déplacement des couches alors que 33\% des schémas montrent une faille sous forme d'un trait rectiligne rayant les strates sans aucun marqueur de déplacement.

Deux schémas ont retenu notre attention par leur présentation de la faille (Figure 4). Ainsi, on retrouve sur le schéma S12 quatre traits rectilignes 
parallèles de même taille et de même épaisseur qui correspondent à des failles inverses. Le jeu de ces failles est marqué en légende mais il n'est pas reflété sur le schéma et ne montre aucun marqueur de déplacement notamment le sens de déplacement et le rejet.

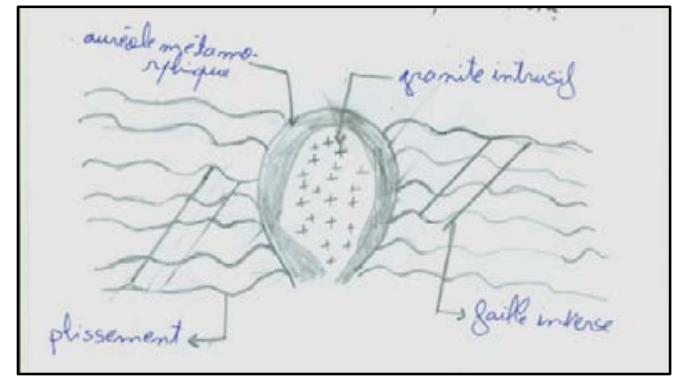

S12

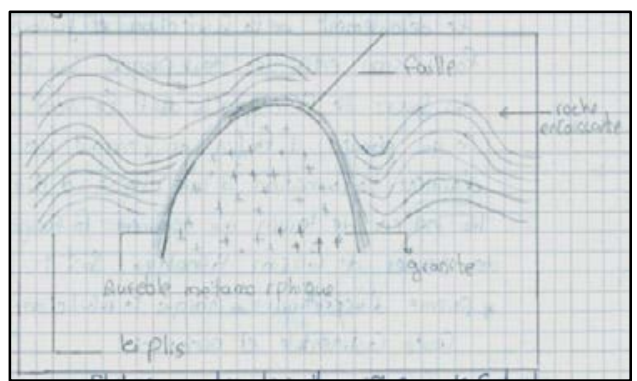

S11

Figure 4 : Exemples de représentation de la faille dans les schémas des enseignants stagiaires

Sur le schéma S11, la faille est schématisée sous forme d'un trait rectiligne montrant du vide de part et d'autre de la faille. Ceci nous incite à penser à une lacune de compréhension : l'enseignant stagiaire ne sait pas comment représenter les strates sédimentaires de part et d'autre de cette faille, c'est pourquoi on suppose qu'il marque un vide.

\section{L'élément 3 : Le plissement et les roches encaissantes}

Notre troisième élément à analyser est le plissement auquel nous avons associé les roches encaissantes. Ceci a révélé que les plissements sont schématisés pour la plupart des enseignants stagiaires comme de grandes structures ondulées marquant des anticlinaux ou des synclinaux, droits ou déversés de différentes tailles. Cependant d'autres schémas marquent des formes diversifiées et qui montrent des plis comme de petites ondulations de très faibles amplitudes sous forme de vagues à lignes discontinues (Figure $5, \mathrm{S9}$ ) ou continues très fines et parfois très serrés (S14). Concernant les figurés marquant les roches encaissantes $53,33 \%$ des schémas montrent des strates à figurés marquant les différents types de roche, alors que sur 46,67\%, ces figurés sont absents et les différentes strates sont représentées sous forme de bandes vides à l'intérieur sans aucun figuré qui pourrait montrer la nature de la roche encaissante (S9). 


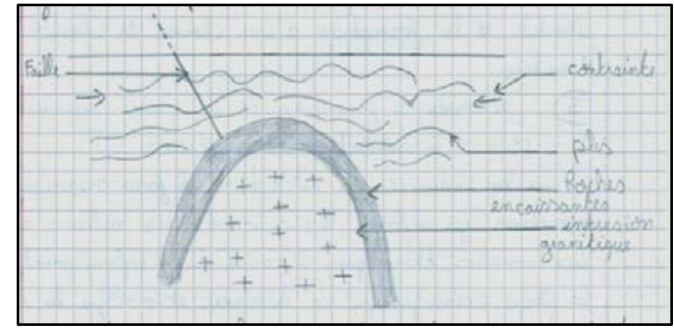

S9

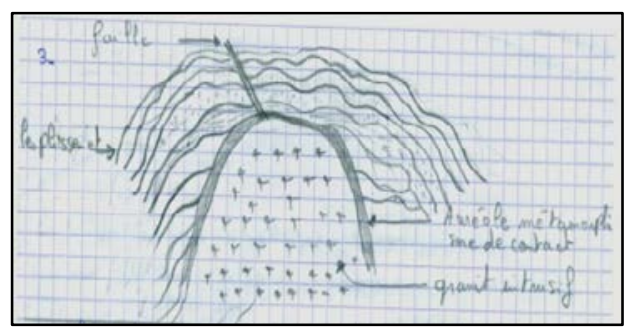

S14

Figure 5 : Quelques représentations des plis sur les schémas des enseignants stagiaires

Le schéma S9 montre, en plus, des flèches schématisées de part et d'autre du pli et qui indiquent les forces de compression. On note que c'est le seul schéma qui montre des forces de compression. Le positionnement de ces flèches à ce niveau marque une contrainte compressive causant une déformation souple affectant les roches encaissantes. L'enseignant stagiaire doit comprendre la signification des deux flèches et la raison de leur positionnement à ce niveau exact.

Dans les schémas analysés, l'intention de l'enseignant stagiaire est de décrire et d'expliquer quelques concepts de base de la géodynamique interne et d'agencer spatialement les données pour faciliter la compréhension et matérialiser sa pensée, pour exposer la manière dont il visualise le contexte global de ces concepts clés. Toutefois même si les productions présentent parfois des lacunes et manquent de finesse et de soin, nous retrouvons bien tous les éléments du schéma.

Ainsi, d'après l'analyse effectuée des éléments clés du schéma nous constatons que les éléments principaux qui sont le granite, la faille, les plis et la roche encaissante sont bien reconnaissables et sont représentés de manière simplifiée quoi qu'ils manquent de précision facilitant la lecture du schéma et la reconnaissance exacte de chaque élément. Aussi les différents éléments schématisés sont visibles et bien lisibles. Ceci montre clairement que pour la majorité des enseignants stagiaires la compréhension de ces éléments clés est simple mais leur représentation schématique présente des difficultés. On peut dire que la plupart des enseignants stagiaires ont pris soin de leurs productions et sont arrivés à présenter un schéma clair et simple. En revanche, des différences nettes apparaissent entre les schémas dans les traitements les plus globaux des informations. Si certains enseignants stagiaires y sont arrivés spontanément, d'autres auront besoin d'être guidés.

\section{L’organisation méthodologique}

Certains aspects d'un schéma sont repérés, parce que leurs symboles sont disposés dans l'espace selon une certaine façon ou associés à des dispositifs graphiques tels que lignes ou démarcations (Lowe, 1996). Dans ce 
contexte, nous étudions sous cette rubrique l'organisation des éléments à schématiser : le granite, la faille, le plissement et leur positionnement les uns par rapport aux autres.

L'analyse des schémas a montré que $70 \%$ des enseignants stagiaires ont bien compris les consignes données dans le test et ont bien essayé de respecter la chronologie des données, ce qui est bien reflétée sur leurs schémas. Alors que dans 30\% des schémas, les éléments essentiels sont bien présentés mais l'organisation et l'agencement entre eux n'est pas bien montré.

Le schéma représenté dans la figure 6 montre deux ensembles différents, posés loin l'un de l'autre : un ensemble à terrains plissés et faillés et un deuxième ensemble, représenté par le granite, est marqué par une structure en dessous des autres et sans aucun lien avec le reste du schéma. On remarque l'existence des traits fins obliques qui remplissent le vide existant entre ces deux ensembles. Ce schéma est lacunaire, on a l'impression que les formations flottent. Notons que chaque ensemble est schématisé d'une manière claire et soignée: le plissement est bien représenté ; la faille inverse est marquée par le déplacement des strates qui sont mis en valeur par des figurés bien illustrés. Enfin, le granite et sa limite marquant l'auréole du métamorphisme sont bien mis en valeur.

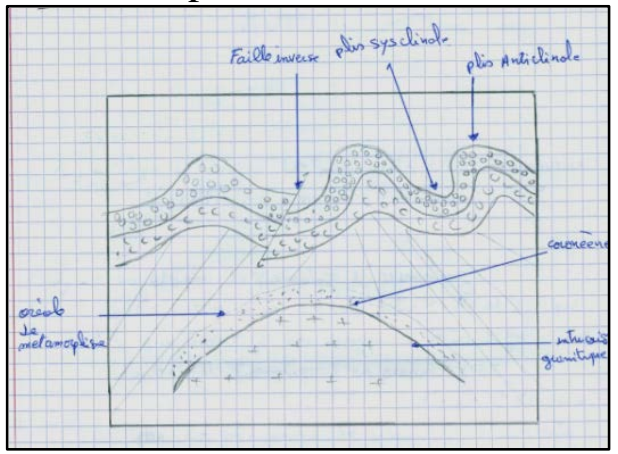

Figure 6 : Exemple de schéma (S26) montrant l’organisation des événements géologiques

Nous notons que sur le S28 (Figure 1), on retrouve aussi deux ensembles représentés mais moins bien marqué. Le schéma montre une ligne fine ondulée qui traduit peut-être la continuité des plis sauf que son positionnement loin des strates plissées laisse penser à une couverture ou à l'existence d'un toit.

Alors que dans la majorité des schémas on a l'impression que l'élément granite est le principal élément représenté, les représentations S23 et le S25 (Figure 7) montrent les éléments (granite, faille, plis et roches encaissantes) avec un même degré d'importance. La représentation de ces éléments montre qu'aucun objet n'est primordial par rapport à l'autre ; ils appartiennent tous à un même ensemble, et ne montrent aucun espace entre 
eux. Dans ce sens Lowe (1989) a montré la nécessité d’une indication supplémentaire explicite dans un schéma quant à l'accentuation graphique des différents éléments du schéma et leur disposition spatiale car, selon cet auteur, ce qui est parfaitement clair pour le producteur de schéma peut être tout à fait opaque pour le lecteur.

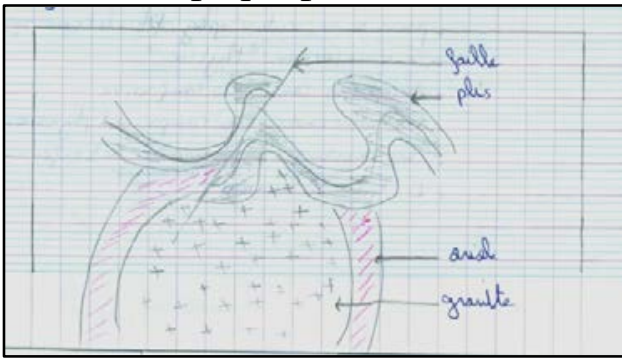

S23

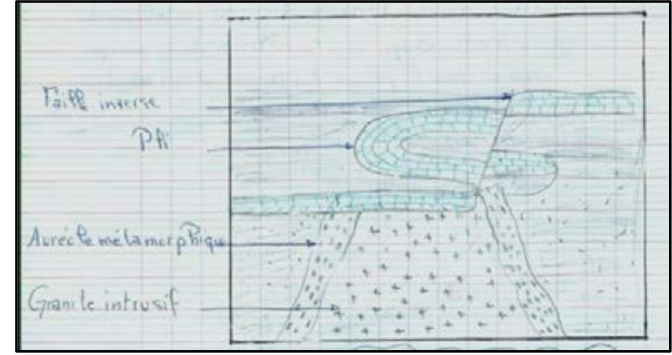

S25

Figure 7 : Exemple de représentation schématique du granite, faille et plis en un seul bloc

Ainsi, sur le plan organisation méthodologique, les enseignants stagiaires ont globalement respecté la consigne imposée par le test à savoir la chronologie des données et l'ont bien reflété sur leur schéma. Cependant, cette organisation pose quelques problèmes puisqu'on s'est retrouvé avec des éléments qui sont présents mais l'organisation et l'agencement entre eux n'est pas bien montré et il n'y a pas de lien qui raccorde les différents éléments. Aussi, il ressort de l'analyse de nos schémas que le granite est l'élément qui est perçu en premier avant les autres en raison de ces caractéristiques visuelles spécifiques, avec son symbole, sa grandeur, l'épaisseur de son trait, sa nuance, sa couleur, sa forme ou encore sa dimension; aussi il a été soigné plus que les autres éléments. On sent que c'est l'élément clé du schéma. Cependant, certaines productions des enseignants stagiaires, montrent qu'ils ont une image assez floue de certains concepts scientifiques et le caractère imaginaire est notable sur certains schémas.

\section{L'usage du code symbolique}

La recherche d'information dans les schémas, repose sur une connaissance des systèmes symboliques et des conventions de schématisation, c'est pourquoi on a tenté d'analyser le langage symbolique des schémas, utilisé par les enseignants stagiaires à savoir le respect des éléments qui constituent un schéma qui sont le titre, la légende, le cadre...

Une présentation soignée reste relative; mais nous entendrions par une présentation soignée celle qui est d'abord faite par un crayon, qui présente un cadre, un titre et une légende. En réalité, l'utilisation de la légende est commune à un grand nombre de productions des enseignants stagiaires, ainsi $96 \%$ des schémas présentent une légende. Cependant, cette 
légende est dans la plupart du temps mal présentée comme le montre le schéma S13 (Figure 8) où les flèches sont mal organisées, dirigés dans des sens différents et qui indiquent une notation de la légende sans aucun arrangement.

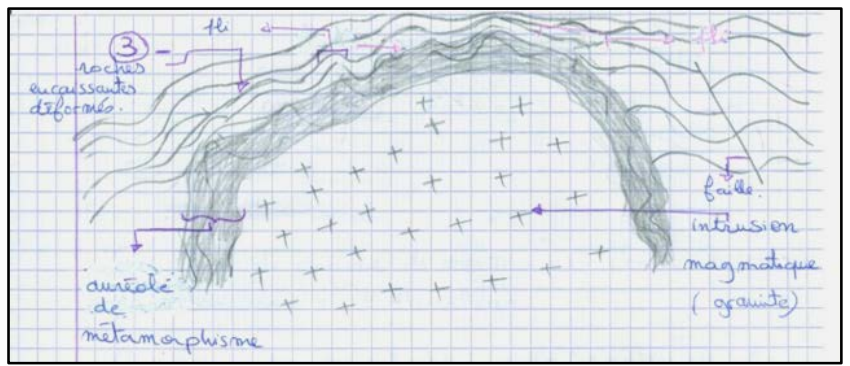

Figure 8 : Exemple de schéma (S13) montrant une légende mal organisée

Concernant le titre, $80 \%$ des productions présentent un titre. Ce dernier fait référence à différents éléments du schéma (Figure 9), ainsi on retrouve sur $37,50 \%$ de schémas : la mise en place du granite ou le granite intrusif, $25 \%$ de schémas évoquent en plus du granite des roches encaissantes, $16,66 \%$ des schémas évoquent le granite avec les contraintes, la faille ou le plissement, 12,50\% des schémas évoquent un métamorphisme de contact et 8,33\% des schémas lient ce métamorphisme de contact au plissement et à la faille.

\section{Pourcentage des éléments clés du titre}

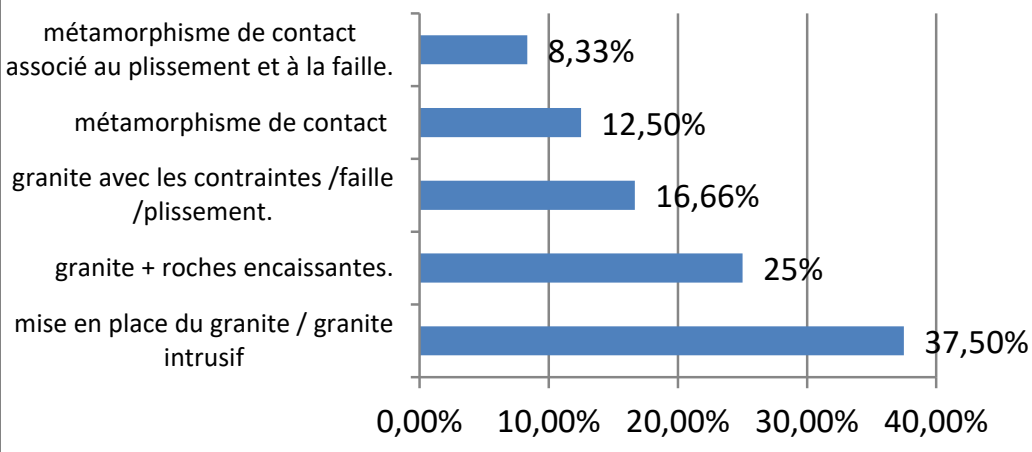

Figure 9 : les titres énumérés sur les schémas des enseignants stagiaires

Quoi que certains schémas ne sont pas bien soignés, l’analyse globale du langage symbolique utilisé par les enseignants stagiaires montre que ces derniers ont essayé de donner une vue correcte de leur représentation : l'usage du code symbolique est fortement respecté à savoir la présence d'un titre, d'une légende et d'un cadre. Ainsi, les différents éléments du schéma sont légendés et sont reliés à leurs noms avec des flèches. Ce type de fléchage témoigne d'une première organisation de l'information écrite 
(Cooper et Destin, 2015), or dans la majorité des schémas ce système de fléchage n'est pas bien travaillé.

Les titres attribués aux schémas appuient l'idée que le granite est l'élément qui ressort par rapport à ses voisins puisque la majorité des schémas présentent dans leur titre la mention du granite seul ou en notant son impact sur les roches encaissantes.

Aussi, les productions analysées permettent d'observer que certains enseignants stagiaires utilisent intuitivement des éléments de schématisation. Le code scientifique mis en place doit être fait sur des bases scientifiques. Cependant, sur les schémas étudiés, ces annotations permettent de nommer les éléments présents, mais n'expliquent ni ce qu'ils sont ni leurs fonctions respectives. Ces annotations sont censées préciser le nom des concepts schématisés et être un support pour la compréhension (Giot et Quittre, 2008).

\section{Corrélations des schémas avec les profils des enseignants stagiaires}

En analysant les profils et le parcours académique des enseignants stagiaires, on peut noter que nous ne pouvons pas trancher sur une liaison entre le type de licence obtenue et les capacités acquises dans le domaine de la communication schématique. Mais, la corrélation de ces schémas avec les profils des enseignants stagiaires nous a permis de noter certains points liés à leurs connaissances :

- 36,66\% des apprenants ont une licence professionnelle ou fondamentale en biologie ou en écologie, obtenue entre 2012 et 2014. Quoi que ces enseignants stagiaires ont approfondi leur connaissance par l'obtention d'une deuxième licence LPQME, ils ont gardé des lacunes en géologie qui seraient peut-être dues à leur spécialisation en biologie;

- $\quad$ les enseignants stagiaires qui ont une licence en filière universitaire éducative représentent 36,67\% (11 FUE) et sont spécialisés en SVT. Leur formation en cette filière éducative leur a permis d'acquérir des capacités des nouvelles technologies; des connaissances du métier de professeur; des outils didactiques mais il leur manque un approfondissement de leurs connaissances scientifiques qui est bien reflété sur leurs représentations schématiques. Ceci pourrait être dû au temps alloué à cette formation et à la nature des thèmes abordés en cette licence.

- $\quad 20 \%$ des enseignants stagiaires ont une Licence fondamentale en géologie obtenue entre 2002 et 2012 et qui ont tous obtenus par la suite une nouvelle licence LPQME et pour certains d'entres eux, un master en géologie. Les représentations schématiques de ces enseignants stagiaires quoi qu'elles présentent quelques erreurs mais sont, en général, plus structurées, bien soignées et claires avec un respect de l'usage du code symbolique notamment une légende, un cadre, des symboles... 
- $\quad$ enfin, des enseignants stagiaires qui ont une licence fondamentale en biologie obtenue en 2015, et qui ne représentent que 6,67\% ont pu garder des lacunes en données géologiques vu leur spécialisation en biologie.

Ainsi, ces enseignants stagiaires même s’ils ont reçu une formation académique et universitaire concernant la géologie et précisément la géodynamique interne au cours de leur cursus scolaire et lors de leur formation universitaire ont des difficultés d'appliquer leurs pré-acquis dans une représentation schématique.

\section{Conclusion, recommandations}

L’analyse faite au niveau des schémas des enseignants stagiaires en SVT, de certains concepts de la géodynamique interne témoigne d'une maitrise moyenne des concepts scientifiques et d'une difficulté de leur représentation en un schéma scientifique simple, lisible et facile à interpréter.

Un certain nombre de recherches ont montré que les étudiants de tout âge éprouvent des difficultés à lire et à utiliser les représentations graphiques (Tiberghien, 2002). Dans notre cas les schémas recueillis des enseignants stagiaires permettent de mettre en évidence des obstacles majeurs liés à la vision spatiale des concepts et à leur représentation dans un schéma clair. Ces obstacles se manifestent principalement par :

- $\quad$ une attribution d'une forme scientifique au schéma, par respect du code symbolique et par la représentation de l'essentiel d'une manière simple sans ambiguïtés pouvant altérer son interprétation ou sa compréhension ;

- $\quad$ une mise en valeur du lien entre les différents éléments du schéma en les disposant convenablement les uns par rapport aux autres selon les contraintes imposées au test ;

- $\quad$ une présence des lacunes sur les schémas qui posent des problèmes de compréhension telle que nous l'avons constaté, à savoir des lacunes au niveau des limites de la faille, des roches encaissantes ou dans l'ensemble des éléments représentés. Nous retenons que ces apprenants en gardant des lacunes sur leur schéma ne signifie pas qu'ils n’ont pas l'information exacte mais ceci peut être lié à une difficulté d'imaginer l'agencement des différents éléments dans l’espace.

Ces difficultés pourraient être liées au fait que ces enseignants stagiaires ne sont pas habitués à la schématisation sur papier, en effet durant leur parcours scientifique, l'utilisation des ressources numériques comme moyen didactique a remplacé leur capacité de schématisation. Ceci a induit que nos stagiaires ne maitrisent pas parfaitement l'art de représenter les données scientifiques dans un schéma. A cela on pourrait ajouter un manque de vocabulaire scientifique dû principalement aux problèmes de la langue d'apprentissage (le passage de l'arabe au niveau scolaire à des études 
universitaires en français) qui se manifeste par la présence des noms erronés notés sur les schémas, et à la non maitrise des concepts géologiques due au type de licence obtenue (biologie, écologie, FUE...).

Apprendre à utiliser des représentations schématiques en sciences est propice aux apprentissages. Toutefois, ce travail nécessite une formation particulière qui permette à l'apprenant de s'approprier des codes de fonctionnement tant en situation de production que de réception. Ces enseignants stagiaires sont censés transmettre le savoir à leurs élèves à travers des schémas bien présentés et bien légendés, c'est pourquoi ils doivent acquérir des capacités pour réussir un schéma scientifique, pouvoir le transmettre et l'expliquer. Dans ce sens la formation initiale complétée par celle qualifiante au métier d'enseignant joue un rôle primordial dans l'acquisition de ces compétences par les enseignants. C'est pourquoi, il nous semble à la lumière de ces résultats qu'il est nécessaire, lors de la formation qualifiante des enseignants, au niveau des CRMEF de :

- $\quad$ allouer plus de temps à la matière des SVT par des cours approfondis accompagnés de travaux pratiques qui permettraient aux enseignants stagiaires de maitriser les concepts avant leurs schématisations puisque comme le montrent Giot et Quittre (2011), la réalisation d'un schéma doit se baser sur des connaissances exactes des concepts à schématiser ;

- $\quad$ proposer des séances ou les enseignants stagiaires pourraient réaliser un schéma afin qu'ils, d’une part, dépassent leurs représentations initiales et, d'autre part, s'approprient les codes usuels de ce type d'écrit. Pour ce faire, nous orientons notre démarche de la façon qui placera les stagiaires dans des situations de formation apprentissage qui les encouragent à utiliser le schéma dans leurs pratiques éducatives. Enfin leur permettre d'apprécier l'évolution de leurs représentations initiales par rapport au travail mené durant les séquences de formation.

- $\quad$ inciter les futurs enseignants à utiliser les schémas dans leur leçon au niveau de leur futur travail et de s'attarder sur l'apport didactique d'un schéma dans une séquence pédagogique. L'encouragement de nos enseignants stagiaires à produire leurs propres schémas au cours de la formation va non seulement les aider à acquérir des capacités de schématisation mais aussi une facilité d'explication de concepts scientifiques ; une vision spatiale...

- $\quad$ attribuer à ce type de communication le temps nécessaire pour sa maitrise afin de transférer ces acquis aux élèves et de ne pas supposer que les conventions utilisées dans un schéma sont forcément connues et comprises.

- $\quad$ ne pas abuser de l'utilisation de ressources numériques aux dépens de schémas construits. 


\section{References:}

1. Astolfi J.P, Develay M. (1989). La didactique des sciences. Paris : PUF, Que sais-je ?. pp. 31-42, 80-85.

2. Astolfi J.P., Darot E., Ginsburger-Vogel Y., Toussaint J. (1998). Mots-clés de la didactique des sciences : Repère, définitions, bibliographies. Bruxelles : De Boeck Université. pp. 45-62, 139-164.

3. Astolfi, J.P., Peterfalvi, B. et Vérin A. (1998 rééd. 2001). Comment les enfants apprennent les sciences ? Paris : Retz. 267p.

4. Bultez A. (2012). Le schéma peut-il être une aide chez l'élève en difficulté dans la résolution de problèmes. Mémoire Education. 45p.

5. BUTY, C., BADREDDINE, Z., (2009). Quelques effets didactodiscursifs de l'utilisation des schémas. Aster. Pp. 48, 89-110.

6. Chantal, M. (2010). Représenter à l'école : dessin ou schéma ? http://lewebpedagogique.com/sciencesalecole/files/Du-dessin-ausch\%C3\% A9ma-\% C3\%A0-1\%C3\%A9cole- primaire1.pdf

7. Cooper I., Destin M., (2015). La schématisation en sciences à l'école élémentaire. Mémoire Education. 62p.

8. Denis 1982. Représentation imagée et résolution des problèmes, revue française de pédagogie, 60, pp.19-29.

9. Giot B., Quittre V., (2011). Lire et produire les schémas scientifiques. Lettrure $\mathrm{N}^{\circ} 1$ pp. 82-92.

10. Giot B., Quittre V. (2008). Images, dessins et schémas scientifiques : comment sont-ils perçus par les enfants ? Cahiers des sciences de l’éducation. Université de Liège (ASPE)-27-28. pp. 125-150.

11. Giot B., Demonty I., Quittre V., (2006): Comprendre et utiliser l'écrit dans les activités scientifiques en $5 e$ et $6 e$ années primaires $a S P e$, Université de Liège.

12. Langellier B. (2004): Quelle différence entre schéma et dessin ? Repéré à http://www.fondation-lamap.org/fr/topic/13497.

13. Leplat J., Hoc J.M, (1983): Tâche et activité dans l'analyse psychologique des situations, Cahiers de psychologie cognitive, 1983, 3, n 1, 49-63.

14. Lowe R., (1996): Les nouvelles technologies, voie royale pour améliorer l'apprentissage des sciences par l'image. Aster $\mathrm{N}^{\circ} 22$, 1996, Images et activités scientifiques, INRP, pp. 173-194.

15. Ministère de l'éducation nationale (MEN) (2009) : Programme et orientations pédagogiques en sciences de la vie et la terre. Cycle collégial. Direction du currucula et de la vie scolaire.

16. Monnier N., (2003). Les schémas dans les activités de résolution de problèmes ; Grand N, n 71, pp. 25 à 47

17. Oillic M.C., (2014). Le schéma scientifique au Cycle 3, Mémoire Education. 62p. 
18. Peraya D., (1995). Vers une théorie des paratextes : images mentales et images matérielles. Recherche en communication, 4, 1-38.

19. Peraya D., \& Nyssen M.C. (1995). Les paratextes dans les manuels scolaires de biologie et d'économie. Une étude comparative. Université de Genève : Cahier $n^{\circ} 78$.

20. Pollard F, Les représentations en sciences: Dessin, croquis ou schéma. www.ac-grenoble.fr/ien.bv/.../doc_Les_representations_Dessin_croquis_schema.doc

21. Vezin J.F, (1986). Schéma et acquisition des connaissances. In: Revue française de pédagogie. Volume 77. pp. 71-78. 